\title{
FORMULATION AND IN VITRO SKIN PENETRATION OF A SOLID LIPID NANOPARTICLE GEL CONTAINING COFFEA ARABICA EXTRACT
}

\author{
JIHAN AGEIS, HERMAN SURYADI, MAHDI JUFRI*
}

Laboratory of Pharmaceutics and Pharmaceutical Technology Development, Faculty of Pharmacy, Universitas Indonesia, Depok, Indonesia. Email: mahdi.jufri@farmasi.ui.ac.id

Received: 20 September 2019, Revised and Accepted: 28 January 2020

\section{ABSTRACT}

Objective: The extract of Coffea contains caffeine that could be used for its anticellulite activity. This study aimed to formulate a Coffea arabica grounds residue extract into a solid lipid nanoparticles (SLNs) gel dosage form and examine the physical stability and in vitro skin penetration of the formulation.

Methods: Coffee grounds residue (CGR) extracts were made into three SLN formulations with different glycerin monostearate (GMS) concentrations of $1 \%, 2 \%$, and $3 \%$. The SLN F2 formulation was a gel created by high-pressure homogenization (HPH). The in vitro penetration assessed using Franz diffusion cells and the physical stability of the SLN extract gels was compared with those of the nonsense extract gel.

Results: Formulation F2 with $2 \%$ GMS had a mean particle size (PS) of $60.3 \mathrm{~nm}$, a polydispersity index (PDI) of 0.278 , and zeta potential of $-32 \pm 1.40$. The PS for the SLN gel after HPH was $159 \mathrm{~nm}$ and the PDI was 0.211 . Cycling and mechanical tests showed that the SLN gel was physically stable. The cumulative amount of caffeine penetrated in vitro was $5.55 \pm 0.08$ for the CGR-SLN gel and $4.18 \pm 0.08$ for the CGR gel.

Conclusions: The amount of caffeine penetrated into rat skin was greater for the CGR-SLN gel than for the CGR gel.

Keywords: Coffea arabica, Microwave-assisted extraction, Solid lipid nanoparticle, Gel, Solid lipid nanoparticle gel, Physical stability.

(C) 2020 The Authors. Published by Innovare Academic Sciences Pvt Ltd. This is an open access article under the CC BY license (http://creativecommons. org/licenses/by/4. 0/) DOI: http://dx.doi.org/10.22159/ijap.2020.v12s1.FF040

\section{INTRODUCTION}

Modern big cities have many coffee shops that produce coffee grounds residue (CGR) waste. This waste is mostly discarded and only a small portion has been used as fertilizer. CGR contains organic compounds, such as fatty acids, amino acids, polyphenols, minerals, and polysaccharides [1]. One of the active substances in CGR is caffeine, which is a methylxanthine compound classified as an alkaloid. Caffeine is a stimulant but also shows anticellulite activity. The topical administration of caffeine through the hydrophilic stratum corneum $(\log \mathrm{p}=-0.07)$ is difficult. Therefore, a delivery system that can enhance the penetration of caffeine through the skin would be useful $[2,3]$.

Solid lipid nanoparticles (SLNs) are submicron colloidal carriers composed of physiological lipids (range of submicron sizes: 50-1000 $\mathrm{nm}$ ) that are usually dispersed in water or an aqueous surfactant solution [4]. Lipid nanocarriers have many advantages for topical application [5] such as the ability to enhance drug penetration into specific skin layers due to their nanosize, the feasibility of incorporating lipophilic and hydrophilic drugs, and less toxicity because physiological lipids are used $[2,4,6]$. SLNs are typically used as a carrier in semisolid formulations, such as gels and creams [7], and can be used to formulate active natural compounds. This study aimed to formulate a CGRs extract of Coffea arabica into SLNs gel dosage form and examine the physical stability and in vitro skin penetration of the formulation.

\section{METHODS}

Materials

The following materials were used in this study: CGR extract, standard caffeine (Sigma-Aldrich, US); Carbomer 940, methylparaben, propylparaben, triethanolamine, propylene glycol, Tween 80 , glycerin monostearate (GMS), distilled water, methanol, ethanol (Brataco Chemical, Indonesia), and Phospholipon ${ }^{\circledR}$ 90G (Lipoid, Germany).
Microwave-assisted extraction

The microwave-assisted extraction method was based on a method with modifications used in the previous study [8]. Ten grams of CGR and $200 \mathrm{~mL}$ of distilled water were placed in a beaker, and the mixture was homogenized for $2 \mathrm{~min}$. Then, the beaker was placed in a microwave at a power of $630 \mathrm{~W}$ for $5 \mathrm{~min}$. The mixture was then filtered through Whatman filter paper No. 1. Then, the filtrate was evaporated and the crude extract was obtained.

\section{Caffeine essay in CGR extract}

A high-performance liquid chromatography (HPLC) method was used to assay the caffeine content in C. arabica [9]. First, a caffeine stock solution (1000 ppm) was prepared by dissolving standard caffeine $(100 \mathrm{mg})$ in $100 \mathrm{~mL}$ of ethanol. Then, working standards $(40,60,80$, 100,120 , and $140 \mu \mathrm{g} / \mathrm{mL}$ ) were prepared by diluting to volume the caffeine stock solution with ethanol in $25 \mathrm{~mL}$ volumetric flasks. About $100 \mathrm{mg}$ of caffeine extract was dissolved in $\leq 10.0 \mathrm{~mL}$ ethanol. The standard and sample solution were injected into the HPLC system with the following conditions: $250 \times 4.6 \mathrm{~mm} \mathrm{C18}$, detection at $278 \mathrm{~nm}$, a flow rate of $1 \mathrm{~mL} / \mathrm{min}$ at room temperature, a sample volume of $20 \mu \mathrm{L}$, and mobile phase of methanol:double-distilled water (95:5).

Formulation and preparation of CGR extract-loaded SLNs (CGRSLNs)

The CGR extract-loaded SLNs were prepared using a high-pressure homogenization (HPH) method. First, the CGR extracts were dissolved in ethanol. Then, the lipid phase was prepared by heating the CGR and GMS (solid lipid) to $85^{\circ} \mathrm{C}$ until they melted. For the aqueous phase, surfactants combined with a cosurfactant (Tween 80 with Lecithin 90G) in bidistilled water were heated to $85^{\circ} \mathrm{C}$ separately. Then, the hot aqueous phase was added to the lipid phase at $85^{\circ} \mathrm{C}$ with magnetic stirring at $600 \mathrm{rpm}$. An emulsion was obtained using HPH (24,000 rpm for $15 \mathrm{~min}$ ). Then, the emulsion was sonicated using a probe sonicator 
for $25 \mathrm{~min}$. The SLNs were finally obtained by allowing the hot emulsion to cool to room temperature (Table 1). In all SLN formulations, the ratio of surfactants to cosurfactant was kept constant (Table 1).

\section{Characterizations of CGR extract-loaded SLNs}

Particle size (PS) distribution and zeta potential measurements

The mean PS and polydispersity index (PDI) of the SLNs were measured by photon correlation spectroscopy using the dynamic light scattering technique with a Zetasizer (Malvern, UK). Before the measurement, the sample was diluted in $10 \mathrm{~mL}$ of the dispersion medium (distilled water). Then, the SLNs were placed into a cuvette and the size was measured [10].

\section{Entrapment efficiency (EE)}

The EE of the SLN dispersion was determined by the centrifugation method with modification [11]. To separate the drug that was not entrapped, SLNs were centrifuged at $13,000 \mathrm{rpm}$ for $50 \mathrm{~min}$. The amount of caffeine in the supernatant was analyzed by HPLC as follows: $250 \times 4.6 \mathrm{~mm} \mathrm{C} 18$ column, detection at $278 \mathrm{~nm}$, the flow rate of $1 \mathrm{~mL} / \mathrm{min}$ at room temperature, a sample volume of $20 \mu \mathrm{L}$, and a mobile phase of methanol:double-distilled water (95:5)

The drug EE was calculated as follows:

$$
\mathrm{EE}(\%)=\frac{\text { Mass of drug in SLN }}{\text { Mass of drug added }} \times 100
$$

\section{Gel formulation of CGR extract-loaded SLNs}

The CGR-SLN gel formulation was prepared by dispersing Carbomer 940 and SLNs in distilled water and then homogenizing at a speed of $3000 \mathrm{rpm}$ and triethanolamine to form a gel. Methylparaben and propylparaben were dissolved in propylene glycol and added to the SLN gel. The gels were then homogenized for $15 \mathrm{~min}$. Next, the CGRSLN gel was subjected to HPH ( 450 bar for 1 cycle). The same procedure prepared for non-SLN gel formulation without HPH. The concentrations of the gel formulations are presented in Table 2 .

\section{Evaluation of CGR-SLN ge}

The parameter evaluation of the CGR-SLN gel included an organoleptic test, homogeneity, $\mathrm{pH}$ measurement, and also the assessment of viscosity and flow properties. The organoleptic and homogeneity tests included color, odor, phase separation, and clarity. The $\mathrm{pH}$ was measured using a $\mathrm{pH}$ meter. First, the electrode was calibrated against a neutral buffer standard ( $\mathrm{pH}$ 7.01) and a buffer solution of acidic $\mathrm{pH}$ ( $\mathrm{pH}$ 4.01). The CGR-SLN gel was prepared by dissolving it in bidistilled water $(10 \% \mathrm{~b} / \mathrm{v})$. Then, the electrode was used to measure the $\mathrm{pH}$ of the SLN gel solutions at room temperature (30).

Table 1: CGR-SLNs formulations at various lipid concentrations

\begin{tabular}{llll}
\hline Formulation & F1 (\%) & F2 (\%) & F3 (\%) \\
\hline Coffee ground residue extract (g) & 2.24 & 2.24 & 2.24 \\
Glycerin monostearate (g) & 1 & 2 & 3 \\
Tween 80 (g) & 3 & 3 & 3 \\
Lecithin G90 (g) & 1 & 1 & 1 \\
Double-distilled water (mL) & 100 & 100 & 100 \\
\hline
\end{tabular}

CGR: Coffee grounds residue, SLNs: Solid lipid nanoparticles

Table 2: Gel formulations $(\% \mathrm{w} / \mathrm{w})$

\begin{tabular}{lll}
\hline Formulation & CGR-SLN gel (\%w/w) & CGR gel (\%w/w) \\
\hline CGR-SLN extract (g) & 7 & 2.54 \\
CGR extract (g) & - & \\
Carbomer 940 (g) & 0.5 & 0.5 \\
Propylene glycol (g) & 9.8 & 9.8 \\
Methylparaben (g) & 0.18 & 0.18 \\
Propylparaben (g) & 0.02 & 0.02 \\
Triethanolamine (g) & 0.5 & 0.5 \\
Distilled water (g) & 100 & 100 \\
\hline
\end{tabular}

CGR: Coffee grounds residue, SLN: Solid lipid nanoparticle
The viscosity and flow properties of the SLN gels were measured using a Brookfield digital viscometer, USA. The spindle (TF 96) was rotated at 0.5 , $1,2,2.5,5,10$, and $20 \mathrm{rpm}$. Samples of the SLN gels were allowed to settle for 30 min at room temperature (30) before the measurements were taken.

\section{PS distribution and zeta potential measurements}

The mean PS and PDI of the SLNs were measured by photon correlation spectroscopy using the dynamic light scattering technique with a Zetasizer. Before the measurement, $1 \mathrm{~mL}$ of sample was diluted in 10 $\mathrm{mL}$ of the dispersion medium. Then, the samples were placed into cuvettes, and the sizes were measured.

\section{Stability test of CGR extract SLN gel}

The CGR-SLN gels were stored at $4 \pm 2^{\circ} \mathrm{C}, 30 \pm 2^{\circ} \mathrm{C}$, and $40 \pm 2^{\circ}$ for 12 weeks. Any change in color, form, odor, homogeneity, syneresis, and $\mathrm{pH}$ was observed every 2 weeks. Viscosity and flow properties were measured before and after testing at room temperature $30 \pm 2{ }^{\circ} \mathrm{C}$

The cycling test involving sample storage at $4 \pm 2^{\circ} \mathrm{C}$ and $40 \pm 2^{\circ} \mathrm{C}$ each for $24 \mathrm{~h}$ ( 1 cycle) was performed a total of 6 cycles, and a mechanical test (centrifugation) was performed by centrifuging at a speed of $3800 \mathrm{rpm}$ for $5 \mathrm{~h}$. After the cycling test, the samples were examined before and after centrifugation.

\section{In vitro penetration study}

Sprague-Dawley female rats (2-3 months old) were sacrificed using ether. Skin tissue from the abdomen was taken from the rats after carefully shaving an area and removing the subcutaneous fat layer. The treatment in these test animals has passed the ethical review by the Ethical Clearance Committee of Cipto Mangunkusumo Public Hospital, Faculty of Medicine, Universitas Indonesia, with the protocol number: 18-040464. The test was performed using Franz diffusion cells with a diffusion area of $2.01 \mathrm{~cm}^{2}$ and a $15 \mathrm{~mL}$ compartment volume. In the receptor compartment, a phosphate buffer medium of $\mathrm{pH} 7.4$ was maintained at a $250 \mathrm{rpm}$ stirring speed. Sampling was performed by taking $2 \mathrm{~mL}$ of a solution from the receptor compartment after 15, 30, 60, 120, 180, $240,300,360$, and $480 \mathrm{~min}$. Next, the sample solution was immediately replaced with phosphate buffer $\mathrm{pH} 7.4$ to keep the fluid volume constant. For the calibration curve, $25 \mathrm{mg}$ standard caffeine was dissolved in $25 \mathrm{~mL}$ of phosphate buffer $\mathrm{pH} 7.4$. The $1 \mathrm{~mL}$ mixture was placed into a $10 \mathrm{~mL}$ flask and then adjusted to volume with phosphate buffer $\mathrm{pH} 7.4$ to give a standard solution of $100 \mathrm{ppm}$ caffeine. This standard solution was further diluted to give working standards ranging from 1 to $50 \mathrm{ppm}$ [2].

Then, the caffeine concentration was measured by HPLC method. The samples and standards were injected into an HPLC system under the following conditions: $250 \times 4.6 \mathrm{~mm} \mathrm{C18}$, detection at $278 \mathrm{~nm}$, flow rate of $1 \mathrm{~mL} / \mathrm{min}$ at room temperature, sample volume of $20 \mu \mathrm{L}$, and mobile phase consisting of methanol: $1 \%$ acetic acid solution (50:50) at a flow rate of $1 \mathrm{~mL} / \mathrm{min}$. The cumulative amount and flux of caffeine penetrated were then calculated. Statistical analysis of data was analyzed by Excel $[2,10]$.

\section{RESULTS AND DISCUSSION}

\section{Assay of caffeine in the CGR extract}

Linear regression gave the following caffeine calibration curve equation: $y=45868 x+274407$ with an $R$-value $=0.99934$, which indicated very high linearity.

As shown in Fig. 1, the standard caffeine retention time was $3.122 \mathrm{~min}$ and the mean retention time of the CGR samples was $3.073 \mathrm{~min}$. The caffeine content of the CGR extract was 4.47 .

\section{CGR-SLN gel characterizations}

The SLN characterization results are presented in Table 3.

\section{Evaluation of CGR-SLN gel}

The CGR-SLN gel had a light brown color and a viscosity of $1160 \mathrm{cps}$, and the CGR-SLN gel had a pH of 5.02. Rheological measurements of formula showed thixotropic pseudoplastic flow properties. 
PS distribution and zeta potential measurements

The CGR-SLN gel had a PDI of 1.000 before HPH and a PDI of 0.211 after $\mathrm{HPH}$. The PS of the CGR-SLN gel decreased from $505 \mathrm{~nm}$ to $159 \mathrm{~nm}$ after HPH. The potential zeta for CGR-SLN gel was $-29.5 \pm 1.18 \mathrm{mV}$ before $\mathrm{HPH}$ and was $-20.4 \pm 0.55 \mathrm{mV}$ after $\mathrm{HPH}$.

\section{Stability test of CGR-SLN gel}

The stability test results showed that the CGR-SLN gel was stable at low, room, and high temperatures for 12 weeks. Organoleptic properties, color, and homogeneity did not change. During storage for 12 weeks at different temperatures, the $\mathrm{pH}$ fluctuated in the range of 6.05-5.87. The CGR-SLN gel viscosity increased over 12 weeks $(1250 \mathrm{cps})$. The cycling and centrifugation tests showed that the CGR-SLN gel formulation was stable (Fig. 2)

\section{In vitro penetration study}

The cumulative caffeine penetrations of the CGR gel and CGR-SLN gel are shown in Table 4.

Table 3: Summary of SLN characterization results

\begin{tabular}{lllll}
\hline Sample & DV90 (nm) & PDI & $\begin{array}{l}\text { Potential zeta } \\
\text { (mV) }\end{array}$ & $\begin{array}{l}\text { Entrapment } \\
\text { efficiency (\%) }\end{array}$ \\
\hline F1 & 50.4 & 0.366 & $-0.366 \pm 0.69$ & 45.39 \\
F2 & 60.3 & 0.278 & $-0.2 \pm$ & 70.82 \\
F3 & 169 & 0.656 & $-0.656 \pm$ & 81.10 \\
\hline
\end{tabular}

SLN: Solid lipid nanoparticle, PDI: Polydispersity index

\section{DISCUSSION}

Caffeine has an anticellulite activity [2]. In the CGR-SLN formulation, $2.54 \%$ extracted caffeine was equivalent to $0.1 \%$ caffeine because the nano-PS concentration of caffeine can be used from $0.1 \%$ to produce effects. The CGR-SLN was formulated as a gel to achieve a comfortable application to the skin.

The goal of formulating the CGR extract with SLNs and various lipid concentrations was to obtain the best PS because the lipid concentration affects the size of the resulting particles (Table 3) [12]. Increasing the lipid concentration and the amount of fixed surfactant would form a larger PS because the surfactant-covered surface area would not increase. In addition, the increased amount of lipid results in the presence of a lipid surface that cannot be covered by the surfactant, so the lipids will agglomerate and produce a larger PS [13]. The PDI reflects the degree of heterogeneity of particles. The closer the PDI gets to zero, the lower the sample heterogeneity (more homogeneous) PS [14]. PDIs with values $>0.5$ indicate high heterogeneity [15]. Our experiments showed that formulation F2 had a more uniform PS distribution than those of F1 and F3.

Potential zeta measurements in formulas are essential for predicting stability during storage because the measure is an indication of potential instability. Formulation instability, such as flocculation and aggregation, can occur [15]. The zeta potential of the three SLN formulas (Table 3) was in the range of $-30 \mathrm{mV}$, which indicated that the resulting SLN was sufficiently stable [13]. Lecithin has negative charges and will affect the

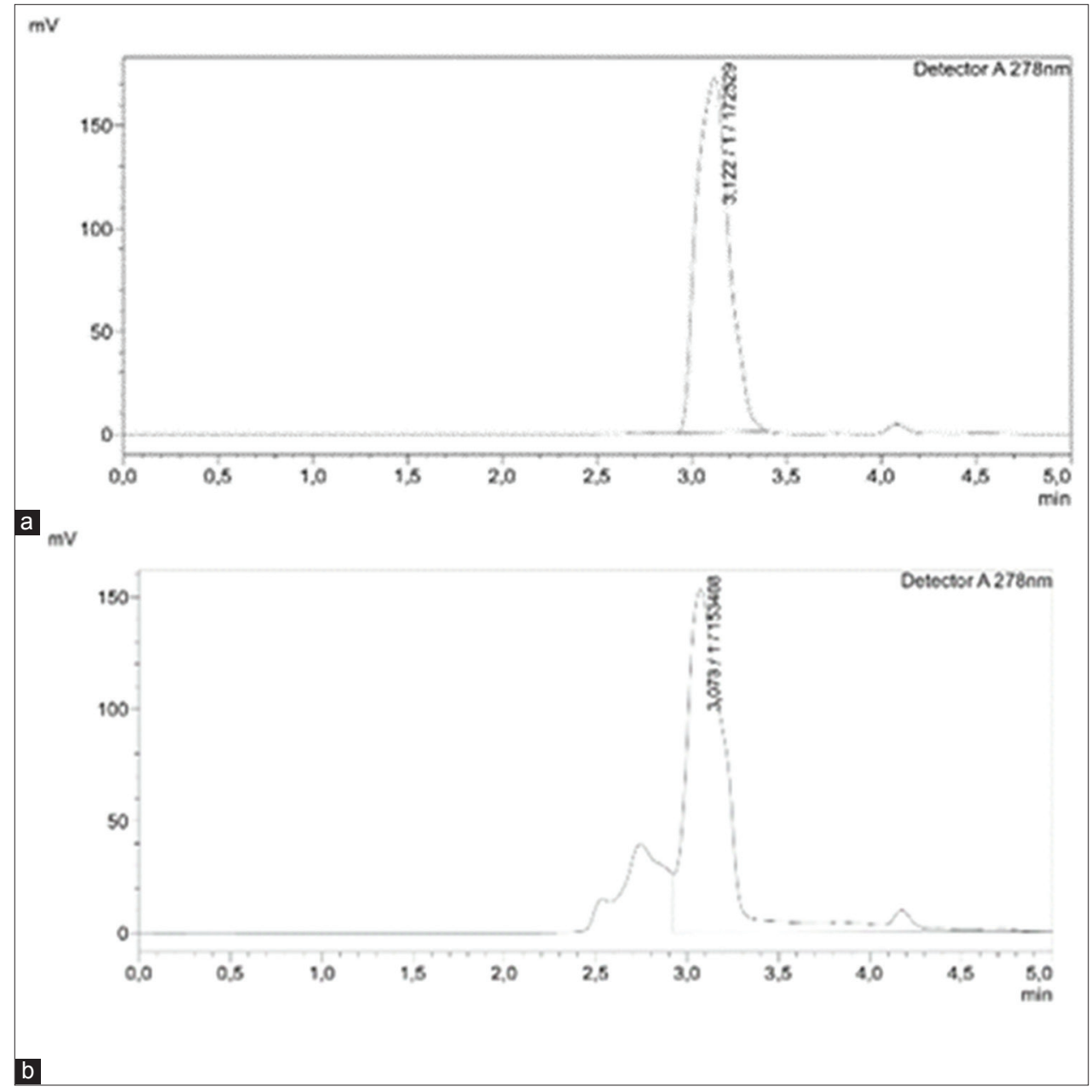

Fig. 1: Chromatograms of (a) caffeine standard solution and (b) coffee grounds residue sample 


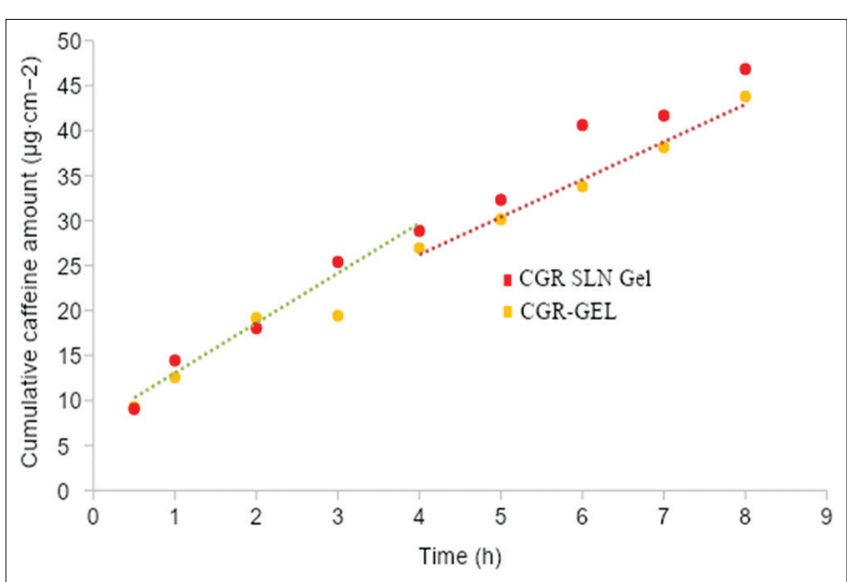

Fig. 2: Cumulative penetration of caffeine from the coffee grounds residue (CGR) gel and CGR-solid lipid nanoparticles gel through rat skin

Table 4: Cumulative caffeine penetration of the CGR gel and CGR-SLN gel

\begin{tabular}{lll}
\hline Formula & Flux value $\left(\boldsymbol{\mu g} \cdot \mathbf{c m}^{-2} \cdot \mathbf{h}^{-1}\right)$ & Average flux $\left(\boldsymbol{\mu g} \cdot \mathbf{c m}^{-2} \cdot \mathbf{h}^{-1}\right)$ \\
\hline CGR gel & 4.11 & 4.180 .08 \\
& 4.15 & \\
& 4.27 & 5.55 \\
CGR-SLN gel & 5.43 & \\
& 5.53 & \\
& 5.59 & \\
\hline
\end{tabular}

CGR: Coffee grounds residue, SLN: Solid lipid nanoparticle

potential zeta value of SLNs, in which the lecithin used comprises $90 \%$ phosphatidylcholine [13].

Regarding the EE of SLNs, Table 3 shows that the higher the concentration of GMS/lipid used, the higher the concentration of caffeine extract is absorbed by the lipid, so the higher the EE. An increased lipid ratio will cause more sites on a drug to be encapsulated by the addition of a surfactant (aqueous phase). Tween 80 will increase the thickness of the hydrophilic coating on the surface of a solid lipid, so drug dispersion increases [16]. In addition to unabsorbed extracts, it may be due to GMS having the capacity to absorb the active substance since the active ingredient added exceeds the lipid capacity. More active substances are not absorbed and have a small EE.

In this study, the selected formulation (F2) had a nano-PS of $60.3 \mathrm{~nm}$, a PDI of 0.278 , a zeta potential of 32 , and an EE of $70.82 \%$, as shown in Table 3. The selected formulation was an SLN gel, which was created using HPH to make the formulation stable. Stability testing showed the SLN gel to be stable under various conditions and to have better drug (caffeine) penetration than that of the non-SLN gel.

The evaluation showed that the CGR-SLN gel had a light brown color due to the dark brown coffee extract that was used to provide the caffeine. The $\mathrm{pH}$ of the CGR extract (pH 5.02) was affected by the pH of the SLN gel. GMS may have affected the pH of the SLN gel due to the stearic acid content. The SLN gel had good viscosity with thixotropic pseudoplastic flow properties. The curved rheogram for pseudoplastic materials resulted from a shearing action on long-chain molecules of materials, such as linear polymers (Tween 80) [17].

The SLN gel before HPH had a PDI of 1.000, which means that the SLN gel was not homogeneous. After HPH, the SLN gel became much more homogeneous, as shown by the PDI of 0.211, and the PS of the SLNs in the gel became smaller, which was related to the number of pressure cycles. The potential zeta of the SLN gel included in the colloidal suspension indicated good stability because it was near $30 \mathrm{mV}$.
Stability testing showed that the SLN gel was stable even though the $\mathrm{pH}$ fluctuated over a narrow range. The $\mathrm{pH}$ decreases observed at room temperature, and the high temperature was due to the susceptibility of polyphenol because of oxidation. In coffee, polyphenols are susceptible to oxidation at $\mathrm{pH} 6-7$ and temperatures above $30^{\circ} \mathrm{C}$ [18]. The changes were acceptable because the observed $\mathrm{pH}$ range was in the acidic $\mathrm{pH}$ range of human skin (4.5-6.5).

The viscosity of the SLN gel had increased at week 12-1250 cps. We concluded that at week 0 after HPH, the molecular structure of the SLN gel was still random due to the high-pressure spin during the process. Still, when stored for 12 weeks, the molecular structure of the SLN gel returned to its original order [16]. The result of cycling and centrifugation test showed that the SLN gel formulation was stable. Because SLN gel did not show changes in physical appearance and syneresis. The gelling agent used in the SLN gel formulation (Carbomer) can retain water in the matrix so that the SLN gel before and after the test remained stable.

As shown in Fig. 2, the CGR-SLN gel gave a greater amount of caffeine penetrated than that of the CGR gel. In this case, the added surfactant acted as a penetration enhancer by dissolving part of the lipids by modifying the stratum corneum integrity [19].

As shown in Table 4, the flux value indicates that the penetration rate of caffeine was faster from the CGR-SLN gel than from CGR gel. The increased penetration of caffeine from the CGR-SLN gel could have occurred through a variety of mechanisms. The CGR-SLN gel was smaller than $200 \mathrm{~nm}$, which meant that the SLNs could pass through the membrane skin to a deeper layer, propylene glycol acted as a penetration enhancer by increasing the fluidity of the lipid bilayer. In addition, the propylene glycol increased the permeability of the caffeine into the stratum corneum [14]. The caffeine of the CGR extract was entrapped in the SLNs that contained lecithin, which has the same structure as that of the lipid bilayer, which was another reason for the increased penetration of caffeine into rat skin [20].

\section{CONCLUSIONS}

Based on the study results, the CGR-SLN gel gave a greater amount of caffeine penetration than that of the CGR gel into rat skin. We expect that the CGR-SLN gel can increase the penetration of caffeine into human skin as well.

\section{ACKNOWLEDGMENTS}

We gratefully thank PITTA grant 2018 from the Universitas Indonesia, who had given a financial support for this research.

\section{CONFLICTS OF INTEREST}

The author has no conflicts of interest to declare.

\section{REFERENCES}

1. Campos-Vega R, Loarca-Piña G, Vergara-Castañeda H, Oomah BD. Spent coffee grounds: A review on current research and future prospects. Trends Food Sci Technol 2015;45:24-36.

2. Puglia C, Offerta A, Tirendi GG, Tarico MS, Curreri S, Bonina F, et al. Design of solid lipid nanoparticles for caffeine topical administration. Drug Deliv 2016;23:36-40.

3. Fadhila M, Mun'im A, Jufri M. A preparation, characterization, and in vitro skin penetration of Morus alba root extract nanoemulsion. Asian J Pharm Clin Res 2019;12:292-6.

4. Müller RH, Radtke M, Wissing SA. Solid lipid nanoparticles (SLN) and nanostructured lipid carriers (NLC) in cosmetic and dermatological preparations. Adv Drug Deliv Rev 2002;54:131-55.

5. Aland R, Ganesan M, Rajeswara RP. Development and optimization of tazarotene loaded solid lipid nanoparticles for topical delivery. Asian J Pharm Clin Res 2019;12:63-77.

6. Müller RH, Mäder K, Gohla S. Solid lipid nanoparticles (SLN) for controlled drug delivery a review of the state of the art. Eur J Pharm Biopharm 2000;50:161-77

7. Sonawane R, Harde H, Katariya M, Agrawal S, Jain S. Solid lipid 
nanoparticles-loaded topical gel containing combination drugs: An approach to offset psoriasis. Expert Opin Drug Deliv 2014;11:1833-47.

8. Upadhyay R, Ramalakshmi K, Rao LJ. Microwave-assisted extraction of chlorogenic acids from green coffee beans. Food Chem 2012;130:184-8

9. Fajara BE, Susanti H. HPLC Determination of Caffeine in Coffee Beverage. IOP Conference Series: Materials Science and Engineering; 2017.

10. Sangsen Y, Likhitwitayawuid K, Sritularak B, Wiwattanawongsa, K Wiwattanapatapee R. Novel solid lipid nanoparticles for oral delivery of oxyresveratrol effect of the formulation parameters on the physicochemical properties and in vitro release. Int J Med Sci Eng 2013;7:873-80.

11. Khazaeli P, Pardakhty A, Shoorabi H. Caffeine-loaded niosomes: Characterization and in vitro release studies. Drug Deliv 2007;14:447-52.

12. Ekambaram P, Abdul HS. Formulation and evaluation of solid lipid nanoparticles of ramipril. J Young Pharm 2011;3:216-20.

13. Mappamasing F, Anwar E, Mun'im A. Formulasi, karakterisasi dan uji penetrasi in vitro resveratrol solid lipid nanopartikel dalam krim topikal (Formulation, characterization and in vitro penetration study of resveratrol solid lipid nanoparticles in topical cream). J Ilmu Kefarmasian Indones 2015;13:137-44.

14. Ramadon D, Wirarti GA, Anwar E. Novel transdermal ethosomal gel containing green tea (Camellia sinensis L. Kuntze) leaves extract: Formulation and in vitro penetration study. J Young Pharm 2017;9:336-40

15. Avadi MR, Sadeghi AM, Mohammadpour N, Abedin S, Atyabi F, Dinarvand $\mathrm{R}$, et al. Preparation and characterization of insulin nanoparticles using chitosan and Arabic gum with ionic gelation method. Nanomedicine 2010;6:58-63.

16. Ghadiri M, Fatemi S, Vatanara A, Doroud D, Najafabadi AR, Darabi M, et al. Loading hydrophilic drug in solid lipid media as nanoparticles: Statistical modeling of entrapment efficiency and particle size. Int J Pharm 2012;424:128-37.

17. Sinko PJ. Physical chemical and biopharmaceutical principles in the pharmaceutical sciences In: Martin's Physical Pharmacy and Pharmaceutical Sciences. Philadelphia, PA: Lippincott Williams and Wilkins; 2011. p. 182-96

18. Mazzafera P, Robinson SP. Characterization of polyphenol oxidase in coffee. Phytochemistry 2000;55:285-96.

19. Bolzinger MA, Briançon S, Pelletier J, Fessi H, Chevalier Y. Percutaneous release of caffeine from microemulsion, emulsion and gel dosage forms. Eur J Pharm Biopharm 2008;68:446-51.

20. Notman R, Anwar J. Breaching the skin barrier--insights from molecular simulation of model membranes. Adv Drug Deliv Rev 2013;65:237-50. 